\title{
Factores de Riesgo Para Desarrollar Empiema Postraumático
}

\author{
Alexander Salcedo, Carlos Alberto Henao, Alexandra Cardona, Santiago Naranjo, \\ María Isabel Villegas, Carlos Hernando Morales
}

\section{RESUMEN}

Introducción: El objetivo de este estudio fue determinar los factores de riesgo asociados con el desarrollo del empiema postraumático en pacientes con trauma de tórax tratados con toracostomía cerrada.

Materiales y métodos: Estudio de casos y controles, en pacientes mayores de 12 años con trauma de tórax que requirieron una toracostomía cerrada para el tratamiento de su lesión torácica. Realizado en el Hospital Universitario San Vicente Fundación, (Medellín, Colombia) entre mayo de 2003 y diciembre de 2010.

Resultados: Se revisaron las historias de 220 pacientes, 52 casos y 168 controles. Se hizo un análisis bivariado y posteriormente una regresión logística en la que se demostró que los Factores de Riesgo (FR) independientes para el desarrollo de empiema postraumático (EPT) fueron: la presencia de hemotórax coagulado postraumático (OR: 52,22 - IC 95\%: 16,99-160,52), no dar antibiótico presuntivo (OR: 4,54 - IC del 95\%: 1,33-15,55) y tener trauma cerrado (OR: 5,07 - IC del $95 \%: 1,48-17,40)$. En la mayoría de los casos, el empiema fue tratado con toracoscopia y toracotomía. El germen más frecuentemente aislado fue el estafilococo aureus.

Conclusión: Dos factores de riesgo independientes son modificables; algunos autores han mencionado la utilización del antibiótico presuntivo para disminuir el riesgo de empiema. Y el hemotórax coagulado postraumático puede ser prevenido verificando tempranamente (posiblemente antes de 48 horas), que espacio pleural esté libre de colecciones.

Palabras claves: Hemotórax retenido, Empiema, Trauma.

\section{ABSTRACT}

Background: The primary objective of this study was to determine the risk factors associated with the development of post-traumatic empyema in patients with chest trauma treated with thoracostomy tube placement.

Materials and methods: Case and control study performed with patients older than 12 years, with chest trauma and who required a thoracostomy tube placement for their treatment. Performed in the San Vicente Foundation University Hospital in Medellin, Colombia, between May of 2003 and December 2010.

Results: The clinical histories of 220 patients were analyzed, 52 cases and 168 controls. A bivariate analysis was conducted and later a logistic regression, which proved that independent risk factors for the development of post-traumatic empyema were: The presence of post-traumatic retained hemothorax (OR: 52.22 - IC 95\%: 16.99-160.52), failure to administrate empiric antibiotic (OR: 4.54 - IC del 95\%: 1.33-15.55) and having a closed trauma (OR: 5.07 - IC del 95\%: 1.48-17.40). In the majority of the cases, empyema was treated with thoracoscopy and thoracotomy. The most frequently isolated pathogen was Staphylococcus aureus.
Conclusion: Two independent risk factors are modifiable; some authors have mentioned the use of empiric antibiotic to diminish the risk of empyema. The post-traumatic retained hemothorax can be prevented by checking early (possibly before 48 hours) the pleural space in search of collections.

Keywords: Retained hemothorax, Empyema, Trauma.

How to cite this article: Salcedo A, Henao CA, Cardona A, Naranjo $\mathrm{S}$, Villegas MI, Morales $\mathrm{CH}$. Factores de Riesgo Para Desarrollar Empiema Postraumático. Panam J Trauma Critical Care Emerg Surg 2012;1(3):163-167.

\section{Source of support: Nil}

Conflict of interest: None declared

\section{INTRODUCCIÓN}

La incidencia de empiema en pacientes con trauma de tórax es del 1 al $4,2 \%^{1,2}$ y alcanza el $8 \%$ en los pacientes que requieren toracostomía. ${ }^{3}$ Puede desencadenar complicaciones como fibrotórax, atrapamiento pulmonar, neumonía recurrente, disminución de la capacidad pulmonar y en algunos casos la muerte, además del aumento en los costos de la atención.

La toracostomía cerrada o la toracocentesis son métodos eficientes para tratar el hemotórax traumático, en la mayoría de los pacientes. Cuando la evacuación es incompleta, se favorecen la inflamación e infección del espacio pleural, produciendo el empiema post traumático (EPT).

La inoculación bacteriana que puede ocurrir durante la inserción del tubo al tórax y el hemotórax retenido, que se presenta entre el 2 y el $25 \%$ de los pacientes con hemotórax traumático, son reconocidos factores asociados al desarrollo del EPT. ${ }^{4,5}$

La identificación temprana de los factores asociados al desarrollo de EPT permite proponer medidas preventivas en los pacientes susceptibles. El propósito de este estudio fue identificar los factores de riesgo para desarrollar EPT en pacientes con trauma de tórax tratados con toracostomía cerrada.

\section{MATERIALES Y METODOS}

Se realizó un estudio de casos y controles con los pacientes mayores de 12 años, que ingresaron al servicio de urgencias del Hospital Universitario San Vicente Fundación (Medellín, Colombia), con trauma de tórax y que requirieron una toracostomía cerrada como medida terapéutica, durante el

Paper presented at the resident competition, 25th Annual Congress of Panamerican Trauma Society, Medellin, Colombia, November 2012. 
periodo comprendido entre mayo de 2003 y 31 diciembre de 2010

Se excluyeron los pacientes con enfermedad crónica debilitante, inmunosuprimidos, trasplantados y quienes tuvieran necesidad de una toracotomía urgente.

Definición de caso: Paciente mayor de 12 años con trauma de tórax, que haya requerido tratamiento con toracostomía cerrada y en quien posteriormente se haya documentado un empiema como complicación postraumática.

Definición de control: Paciente con trauma de tórax, que haya requerido toracostomía cerrada como parte del tratamiento y en quien no se haya documentado empiema como complicación postraumática, durante los treinta días siguientes al tratamiento con toracostomía.

El diagnóstico de empiema se hizo con base en los siguientes criterios: (1) Líquido purulento extraído de cavidad pleural o drenaje del mismo por la sonda de toracostomía. (2) Gram o cultivo positivo para crecimiento bacteriano del líquido extraído de cavidad pleural. (3) Paciente con resultado de un estudio imaginológico (ecografía o TAC del tórax) compatible con empiema. (4) Característica de exudado en el líquido extraído de la cavidad pleural $(\mathrm{Ph}<$ $7,10, \mathrm{LDH}>1000 \mathrm{UI} / 1$ y glucosa $<40 \mathrm{mg} / \mathrm{dl}$ ).

Muestra: Considerando que el hemotórax coagulado postraumático es el principal FR y asumiendo (con base en un trabajo previo) que la incidencia de este FR en pacientes con empiema es del $50 \%$, con un poder del $80 \%$ y un intervalo de confianza del $95 \%$, el tamaño de la muestra se calculó en 67 casos y 201 controles (relación casos y controles 1:3). Se utilizó con el programa EPIINFO 2000. La selección de los casos fue consecutiva, hasta obtener el número total de pacientes con EPT. Los controles fueron los pacientes con las características ya mencionadas que ingresaron durante los siguientes 7 días al paciente caso. Cuando había más de 3 controles por un caso, se escogieron los 3 mas cercanos (en tiempo de ingreso) al caso. Y cuando se encontraron menos de 3 controles, se buscaron en la semana siguiente.

Las variables independientes fueron: Edad, sexo, hemotórax coagulado, número de tubos de toracostomía, duración en días de la toracostomía, mecanismo del trauma (penetrante o cerrado), utilización de ventilación mecánica, ingreso a la Unidad de Cuidado Intensivo (UCI) previo al diagnóstico de empiema, presencia de contusión pulmonar, utilización de antibiótico presuntivo (se consideró unicamente aquel que fue suministrado durante una hora previo a la toracostomía), puntaje revisado de trauma (RTS), tiempo en días de hospitalización en la UCI, germen aislado en el cultivo, tratamiento del empiema (toracostomía, toracoscopia, toracotomía) y días de hospitalización.
Las historias se buscaron en el archivo del hospital, utilizando todos los diagnósticos del CIE-10 relacionados con trauma de tórax y con empiema.

El control de sesgos se hizo mediante la inclusión consecutiva de los casos y el cumplimiento estricto del criterio para seleccionar al control; utilizando preguntas precisas que evitaban interpretaciones subjetivas y entrenando y supervisando a las personas que hicieron la recolección. La base de datos se depuró con pruebas descriptivas (promedios y frecuencias) analizando y revisando los valores máximos y mínimos.

Se hizo un análisis bivariado para establecer la asociación entre cada uno de los factores de riesgo y empiema; este se expresó en OR con sus respectivos intervalos de confianza del 95\%. Posteriormente se hizo una regresión logística para determinar los factores de riesgo asociados de manera independiente con el desenlace; se incluyeron las variables que tuvieron $\mathrm{p}<0,2$ en el análisis bivariado. Las comparaciones estadísticas se realizaron con la prueba U de Mann-Whitney para las variables cuantitativas y Chi cuadrado (Mantel Haensel) y Fisher para las variables categóricas; cuando la $p$ fue menor de 0,05 se consideró significancia estadística. Para la elaboración de la base de datos se utilizó el programa ACCES y para el análisis estadístico, el programa SPSS. ${ }^{19}$

Este estudió cumplió con todas las normas éticas establecidas por los comités de ética de las Instituciones.

\section{RESULTADOS}

Durante el periodo de estudio (mayo de 2003 y diciembre de 2010) se encontraron las historias clínicas de 220 pacientes que cumplieron los criterios de inclusión, 52 casos y 168 controles.

Se evaluaron 14 características como potenciales factores de riesgo para desarrollar empiema postraumático. La frecuencia de presentación de cada factor de riesgo y su asociación con el desarrollo de empiema se presenta en la Tabla 1. Para las variables cuantitativas se evaluó la distribución y se encontró que la edad, RTS, tiempo (días) con toracostomía y, tiempo (días) entre trauma y cirugía, presentaron una distribución no normal (KolmogorovSmirnov $<0,05$ ) en los casos, en los controles o en ambas, por tanto, se hicieron pruebas no paramétricas; el resultado se presenta en la Tabla 2.

Con el análisis multivariado se encontró que los factores de riesgo independientes para desarrollar empiema fueron la presencia de hemotórax coagulado (OR: 52,22 - IC 95\%: 16,99-160,52), no dar antibiótico presuntivo (OR: 4,54 - IC del 95\%: 1,33-15,55) y tener trauma cerrado (OR: 5,07 IC del 95\%: 1,48-17,40) (Tabla 3). El modelo ajusta bien 


\begin{tabular}{|c|c|c|c|c|c|c|}
\hline Variable & $\begin{array}{l}\text { Valores de la } \\
\text { variable }\end{array}$ & $\begin{array}{l}\text { Casos } \\
N: 52\end{array}$ & $\begin{array}{c}\text { Controles } \\
N: 168\end{array}$ & OR & $I C$ & $p$ \\
\hline \multirow[t]{2}{*}{ Edad } & $>0=55$ & 6 & 9 & 2,31 & $0,791-6,926$ & $0,123^{*}$ \\
\hline & $<55$ & 45 & 158 & - & - & - \\
\hline \multirow[t]{2}{*}{ Sexo } & Masculino & 9 & 11 & 2,93 & $1,04-8,25$ & $0,028^{*}$ \\
\hline & Femenino & 43 & 157 & - & - & \\
\hline \multirow[t]{2}{*}{ Tipo Trauma } & Penetrante & 15 & 21 & 2,84 & $1,25-6,43$ & $0,005^{\star *}$ \\
\hline & Cerrado & 37 & 147 & - & - & - \\
\hline \multirow{2}{*}{$\begin{array}{l}\text { Antibiótico } \\
\text { presuntivo }\end{array}$} & $\mathrm{Si}$ & 10 & 51 & 0,546 & $0,254-1,173$ & $0,156^{*}$ \\
\hline & No & 42 & 117 & - & - & - \\
\hline \multirow{3}{*}{$\begin{array}{l}\text { Indicación } \\
\text { toracostomía }\end{array}$} & Neumotórax & 5 & 48 & 1 & - & $<0,001^{* *}$ \\
\hline & Hemoneumotórax & 21 & 92 & 2,19 & $0,72-7,11$ & - \\
\hline & Hemotórax & 19 & 21 & 8,69 & $2,58-31,01$ & - \\
\hline \multirow{2}{*}{$\begin{array}{l}\text { Hemotórax } \\
\text { coagulado }\end{array}$} & $\mathrm{Si}$ & 32 & 15 & 22,55 & $9,9-51,33$ & $<0,001^{*}$ \\
\hline & No & 14 & 148 & - & - & - \\
\hline \multirow{2}{*}{$\begin{array}{l}\text { UCI pre- } \\
\text { empiema }\end{array}$} & $\mathrm{Si}$ & 7 & 3 & 8,55 & $2,1-34,42$ & $0,002^{*}$ \\
\hline & No & 45 & 165 & - & - & \\
\hline \multirow{2}{*}{$\begin{array}{l}\text { Ventilación } \\
\text { mecánica pre- } \\
\text { empiema }\end{array}$} & $\mathrm{Si}$ & 2 & 2 & 3,32 & $0,456-24,17$ & $0,238^{*}$ \\
\hline & No & 50 & 166 & - & - & - \\
\hline \multirow{2}{*}{$\begin{array}{l}\text { Contusión } \\
\text { pulmonar }\end{array}$} & $\mathrm{Si}$ & 7 & 22 & 1,33 & $0,524-3,38$ & $0,616^{*}$ \\
\hline & No & 33 & 138 & - & - & \\
\hline \multirow{2}{*}{$\begin{array}{l}\text { Días con } \\
\text { toracostomía }\end{array}$} & $>0=2$ & 49 & 147 & 2,22 & $0,633-7,80$ & $0,30^{*}$ \\
\hline & $<2$ & 3 & 20 & - & - & \\
\hline \multirow{2}{*}{$\begin{array}{l}\text { Número de } \\
\text { sondas de } \\
\text { toracostomía }\end{array}$} & 2 o más & 11 & 10 & 4,24 & $1,56-11,71$ & $0,002^{*}$ \\
\hline & 1 & 41 & 158 & - & - & - \\
\hline
\end{tabular}

${ }^{*}$ p de Fisher; ${ }^{* *}$ Chi cuadrado de tendencia (Mantel-Haensel)

\begin{tabular}{lccc}
\multicolumn{2}{l}{ Tabla 2: Análisis bivariado de los factores de riesgo para desarrollar empiema. Variables cuantitativas } \\
Variable & Mediana (mínimo-máximo) & $p^{*}$ \\
\cline { 2 - 3 } & Casos & Controles & \\
\hline Edad & $33(16-65)$ & $26(14-75)$ & 0,002 \\
RTS & $7,55(6,1-7,8)$ & $7,55(5,2-7,8)$ & 0,379 \\
Días con toracostomía & $5,97(1,29-17,46)$ & $3,65(0,69-19,49)$ & $<0,001$ \\
Días entre trauma y toracostomía & $0,51(0-16,42)$ & $0,25(0-12,93)$ & 0,009 \\
\hline
\end{tabular}

* Mann-Whitney

\begin{tabular}{lcccc}
\multicolumn{5}{c}{ Tabla 3: Resultado del análisis multivariado para el desarrollo de empiema postraumático } \\
Variables & $B$ & Sig & OR & IC del 95\% \\
\hline Tipo de trauma (cerrado) & 1,623 & 0,10 & 5,07 & $1,48-17,40$ \\
Antibiótico presuntivo (no utilizarlo) & 1,514 & 0,016 & 4,54 & $1,33-15,55$ \\
Hemotórax coagulado & 3,955 & 0,000 & 52,22 & $16,99-160,52$ \\
Constante & $-4,164$ & 0,000 & 0,16 & - \\
\hline
\end{tabular}

Nota: Variables incluidas en el análisis multivariado: edad, indicación para toracostomía (hemotórax, hemoneumotórax, neumotórax), ingreso a Uci previo al diagnóstico e empiema, número de tubos de toracostomía, tiempo con toracostomía y tiempo entre trauma y toracostomía

(Hosmer and Lemeshow: 0,837) y explica el 51,4\% de la variabilidad de la variable dependiente (Nagelkerke: 0,514).

El empiema fue tratado con toracoscopia, en 21 pacientes; con toracotomía, en 20; toracostomía en 4 pacientes; y drenaje percutáneo, en 1 . Nueve pacientes requirieron un procedimiento adicional para el tratamiento de su empiema. En ningún paciente se utilizó trombolíticos intrapleurales.

A 50 de los 52 pacientes con empiema se les hizo cultivo del líquido pleural. Los gérmenes obtenidos fueron: Estafilococo Aureus en 25 (50\%); Estafilococo Aureus 
resistente en 12 (24\%); otros Cocos Gram Positivos en 9 (18\%); y Bacilos Gram Negativos en 4 (8\%).

Debido a que el estudio es retrospectivo hubo pérdida de información, que para los controles fue del 6\%. Para los casos, se perdió el $23 \%$ de la información relacionada con contusión pulmonar; el 11,5\% para hemotórax coagulado e indicación de la toracostomía y para el resto de las variables, fue inferior al $2 \%$.

\section{DISCUSIÓN}

Este estudio es de casos y controles, por tanto no es posible determinar la incidencia de empiema, pero otros autores como Eren y cols, ${ }^{6}$ en un estudio retrospectivo de 2,261 pacientes con trauma de tórax que requirieron toracotomía, informaron la incidencia de EPT en 3,1\% de los pacientes. Recientemente DuBose J y colaboradores hicieron una investigación multicéntrica, aun no publicada, para evaluar la incidencia de empiema entre los pacientes con hemotórax coagulado postraumático e identificar los FR asociados a su desarrollo; encontraron que la incidencia de empiema en estos pacientes alcanza el 26,8\%.

En este estudio, demostró una asociación de gran magnitud entre hemotórax coagulado y EPT después del análisis multivariado. Muchos autores coinciden en que el hemotórax coagulado es un factor de riesgo para el desarrollo de empiema (y DuBose J y colaboradores en su estudio no publicado)..$^{1,5-8}$ Posiblemente el hemotórax retenido, sumado al inóculo bacteriano que puede ocurrir durante la inserción del tubo de toracostomía y la disrupción del tejido pulmonar secundaria al trauma que permite el paso de gérmenes del tracto respiratorio al espacio pleural, facilitan el desarrollo del EPT.

La utilidad del antibiótico presuntivo en trauma de tórax es controvertida. Algunos estudios encuentran que su utilización no es benéfica ${ }^{9-12}$ mientras que otros demuestran que disminuye las complicaciones infecciosas ${ }^{13-16}$ Los resultados son contradictorios, posiblemente porque los tamaños de muestra son pequeños. Un meta-análisis con ensayos clínicos de alta calidad ${ }^{17}$ demostró que el antibiótico presuntivo comparado con placebo, disminuye la incidencia de empiema y de neumonía postraumática; sin embargo existe heterogeneidad entre los estudios, debida a la diversidad de antibióticos y las dosis utilizadas. En este estudio no evaluamos ni la dosis ni el antibiótico utilizado, pero se encontró que, cuando no se utiliza de manera adecuada, es un factor de riesgo independiente para el desarrollo de empiema. La mayoría de los estudios coinciden en que el germen aislado con mayor frecuencia es el estafilococo aureus, por tanto la profilaxis deberá estar dirigida hacia esta bacteria.
La relación entre el tipo de trauma y el empiema varía entre los estudios. La asociación encontrada entre trauma cerrado y empiema, muy probablemente tenga relación con la magnitud del trauma. En el trauma penetrante, el empiema está relacionado con la contaminación directa del espacio pleural; aunque no fue predictor independiente, Eren y $\operatorname{cols}^{6}$ encontraron mayor riesgo de empiema en pacientes con trauma penetrante. En el cerrado, posiblemente estén involucrados otras factores ya descritos como factores de riesgo por otros autores como la contusión pulmonar, ${ }^{6,8}$ las fracturas costales (y DuBose J y cols no publicado) ${ }^{18}$ y con la necesidad de hospitalización en la UCI y ventilación mecánica. ${ }^{6,19}$

Aunque se acepta que un segundo tubo de toracostomía puede ser eficaz para el drenaje del hemotórax traumático, en este estudio, cuando se requiere otro tubo aumenta el riesgo de empiema. Esto se debe, posiblemente, a que en el segundo procedimiento hay posibilidad de un nuevo inóculo bacteriano y es posible que cuando se toma la decisión de ponerlo ya hay un hemotórax coagulado o retenido que debe ser evacuado por métodos diferentes a la toracostomía, por ejemplo por toracoscopia. Un estudio de 584 pacientes con trauma de tórax que requirieron toracostomía identificó la necesidad de múltiples tubos de toracostomía, además de la contusión pleural, como predictores independientes de empiema. $^{8}$

La contusión pulmonar ha sido reportada como factor de riesgo independiente para desarrollar EPT. ${ }^{6,8}$ En este estudio, la pérdida de información sobre este FR específico fue mayor del 15\% lo que impide demostrar alguna asociación.

El tratamiento del empiema es el drenaje del espacio pleural. El método de elección ha sido la toracotomía, sin embargo en las últimas dos décadas, el drenaje por toracoscopia se ha ido imponiendo, con alta probabilidad de éxito si se hace tempranamente (antes de 4 semanas). Independiente de la técnica que se utilice, los principios básicos continúan siendo la evacuación completa del espacio pleural, la reexpansión pulmonar y el control del germen productor de la infección. ${ }^{20}$

La principal limitación de este estudio es la pérdida de información sobre algunas variables, debido a que la recolección de la información se hizo desde las historias clínicas.

\section{CONCLUSIONES}

Coincidiendo con otros autores, en este estudio se demuestra que la presencia de hemotórax coagulado, no dar antibiótico profiláctico y tener un trauma cerrado de tórax, son factores de riesgo independientes para desarrollar empiema post traumático. 
Los dos primeros factores son controlables, pero se requiere el diseño de protocolos de manejo. El primer factor ha sido ampliamente estudiado y se conocen los factores de riesgo para desarrollar hemotórax coagulado, de tal forma que es posible identificar los pacientes de alto riesgo. Es necesario el diseño de protocolos que disminuyan su incidencia y la comprobación de su eficacia. El segundo puede controlarse mediante la administración adecuada de antibiótico preventivo; aunque el germen más frecuente es el estafilococo aureus, se requieren estudios que determinen el antibiótico, la dosis y el tiempo durante el cual debe ser utilizado.

\section{REFERENCIAS}

1. Mandal AK, Thadepalli H, Mandal AK, Chettipalli U. Posttraumatic empyema thoracis: A 24-year experience at a major trauma center. J Trauma 1997;43:764-77.

2. Deneuville M. Morbidity of percutaneous tube thoracostomy in trauma patients. Eur J Cardiothorac Surg 2002;22(5):673-78.

3. Hoth JJ, Burch PT, Bullock TK, Cheadle WG, Richardson JD. Pathogenesis of post-traumatic Empyema: The impact of pneumonia on pleural space infections. Surgical Infections 2003;4:29-35.

4. Sanabria AE. Empiema pleural postraumatico: una entidad diferente. Revista Colombiana de Cirugía. http://encolombia. com/cirugia13498-contenido.htm\#CON.

5. Hoth JJ, Burch PT, Richardson JD. Post-traumatic empyema. Eur J Trauma 2002;28:323-32

6. Eren S, Esme H, Sehitogullari A, Durkan A. The risk factors and management of post-traumatic empyema in trauma patients. Injury 2008;39(1):44-49.

7. Karmy-Jones R, Holevar M, Sullivan RJ, Fleisig A, Jurkovich GJ. Residual hemothorax after chest tube placement correlates with increased risk of empyema following traumatic injury. Can Respir J 2008;15(5):255-58.

8. Aguilar MM, Battistella FD, Owings JT, Su T. Post-traumatic empyema. Risk factor analysis. Arch Surg 1997;132(6):647-50; discussion 650-51.

9. Demetriades D, Breckon V, Breckon C, et al. Antibiotic prophylaxis in penetrating injuries of the chest. Ann R Coll Surg Engl 1991;73(6):348-51.

10. Grover FL, Richardson JD, Fewel BA, et al. Prophylactic antibiotics in the treatment of penetrating chest wounds. J Thorac Cardiovasc Surg 1977; 74:528-36.

11. LeBlanc KA, Tucker WY. Prophylactic antibiotics and closed tube thoracostomy. Surg Gynecol Obstet 1985;160:259-63.

12. Mandal AK, Montano J, Thadepalli H. Prophylactic antibiotics and no antibiotics compared in penetrating chest trauma. $\mathrm{J}$ Trauma 1985; 25:639-43.
13. Evans JT, Green JD, Carlin PE, Barrett LO. Meta-analysis of antibiotics in tuve thoracostomy. Am Surg 1995;61(3):215-19.

14. Brunner RG, Vinsant GO, Alexander RH, et al. The role of antibiotic therapy in prevention of empyema in patients with an isolated chest injury (ISS 9-10): A prospective study. J Trauma 1990; 30:1148-54.

15. Cant PJ, Smart DO. Antibiotic prophylaxis is indicated for chest stab wounds requiring closed tube thoracostomy. Br J Surg 1993; 80:464-66.

16. LoCurto JJ Jr, Tischler CD, Swan KG, et al. Tube thoracostomy antibiotics or not? J Trauma 1986; 26:1067-72.

17. Sanabria A, Valdivieso E, Gómez G, Echeverry G. Prophylactic antibiotics en chest trauma: A meta-analysis of high-qualitystudies. World J Surg 2006; 30(10):1843-47.

18. Flagel BT, Luchette FA, Reed RL, et al. Half-a-dozen ribs: Breakpoint for mortality. Surgery 2005 Oct;138(4):717-23.

19. Luchette FA, Barrie PS. Practice management guidelines for prophylactic antibiotic use in tube thoracostomy for traumatic hemopneumothorax: The EAST practice management guidelines work group. Eastern association for trauma. J Trauma 2000; 48:753-57.

20. Molnar TF. Current surgical treatment of thoracic empiema in adults. Eur J Cardiothorac 2007;32;422-30.

\section{ACERCA DE LOS AUTORES}

\section{Alexander Salcedo}

Residente de Cirugía General, Universidad de Antioquia, Medellín

\section{Carlos Alberto Henao}

Estudiante de Maestría en Epidemiología, Profesor de Cirugía de la Universidad de Antioquia, Medellín

\section{Alexandra Cardona}

Estudiante de Medicina de la Universidad de Antioquia, Medellín

\section{Santiago Naranjo}

Estudiante de Medicina de la Universidad de Antioquia, Medellín Asesores Coinvestigadores

\section{María Isabel Villegas}

Profesora de Cirugía General, Universidad de Antioquia, Medellín

\section{Carlos Hernando Morales (Correspondiente Autor)}

Profesor de Cirugía General, Universidad de Antioquia, Medellín e-mail: alexsalcedo2110@yahoo.es 